\title{
educação

\section{Pensar a Universidade dos próximos 20 anos através de uma metodologia de cenários}

\author{
Isabel AlarcãoI, José Tavares ${ }^{I I}$ \& Óscar Mealha ${ }^{\text {III }}$ \\ Universidade de Aveiro, Portugal \\ Dayse Neri de SouzA ${ }^{\mathrm{IV}}$ \\ Centro Universitário Adventista de São Paulo, Brasil
}

\begin{abstract}
Neste artigo, os autores propõem-se relatar o processo e analisar os resultados de uma abordagem metodológica baseada na construção de cenários para perspetivar a Universidade do futuro nas dimensões da formação, inovação e pesquisa e em função da intensidade valorativa de um conjunto de marcadores: mentes, afetos, autonomia, tecnologias, métodos, organização, edifícios, financiamentos, contextos, equipamentos, comportamentos, empregabilidade, democraticidade e internacionalização, em estudo no âmbito do Projeto "Marcadores de formação, inovação e pesquisa para a Universidade de hoje e do próximo decénio". O presente texto não incidirá sobre a substância da matéria em estudo, ou seja, como é vista a evolução da Universidade no futuro, mas sobre a metodologia de construção de cenários prospetivos em sessões que combinaram uma dinâmica de workshop com a de grupo focal, envolvendo professores, investigadores e estudantes. Analisar-se-ão as dificuldades e potencialidades desta abordagem, as limitações encontradas e os contributos para o estudo global.
\end{abstract}

Palavras-chave: Universidade; Cenários; Grupos focais; Marcadores

\section{CONTEXT UA L I Z A ÇÃ O}

Nestes tempos de incerteza, imprevisibilidade e rápida evolução, como são os que estamos a viver nas primeiras décadas do século XXI, perguntase amiúde o que será a Universidade daqui a uns anos. Numa tentativa de resposta, lançam-se projetos como o "Futures for Higher Education: Analysing Trends"' no Reino Unido, imaginamse cenários como os que a OCDE lançou em 2004 (OCDE/CERI, 2004), analisam-se desafios
(UNESCO, 2003), fazem-se planos estratégicos nas instituições, alguns dos quais vêm a público, como é o caso do da Universidade Federal de Santa Catarina, no Brasil (Moritz et al., 2010).

Pensar a Universidade do futuro e, designadamente, o que esta instituição multisecular poderá vir a ser nos próximos anos é uma questão que começa a colocar-se como um enorme desafio a todos aqueles que se encontram ligados à instituição universitária e que, nessa qualidade, não poderão deixar de prestar especial atenção

\footnotetext{
${ }_{1}$ Disponível em: http://www.universitiesuk.ac.uk/policy-and-analysis/reports/Documents/2012/futures-for-higher-education. pdf (acesso em: 30/05/2016).
} 
à sua (im)previsível evolução, apesar de se encontrarem sempre demasiado ocupados com as tarefas da formação e pesquisa, que hoje se apresentam com uma carga excessivamente burocrática, deixando-lhes pouco tempo para refletir e dialogar.

As sessões de discussão e reflexão sobre esta problemática, que nos propomos descrever neste artigo, tiveram como um dos seus objetivos proporcionar momentos de reflexão conjunta com grupos de professores, investigadores e estudantes universitários, a partir de cenários construídos para o efeito, com base num conjunto de indicadores que, no âmbito do presente projeto, são referidos como "marcadores", previamente identificados e focalizados em três domínios de atividade que se configuram de acordo com a missão da Universidade: formação/educação, inovação, pesquisa/investigação.

Por marcadores deve entender-se uma associação de indicadores ou componentes (variáveis) em rede que, de acordo com a importância ou valor que lhe forem atribuídos, pode determinar a estrutura e a dinâmica da Universidade do presente e do futuro. Trata-se de uma metáfora transposta da ideia de marcador somático de Damásio, Tranel, e Damásio (1991) e Damásio (1994). Esses marcadores, cujos descritores apresentaremos mais abaixo, são os seguintes: mentes, afetos, autonomia, tecnologias, métodos, organização, edifícios, financiamentos, contextos, equipamentos, comportamentos, empregabilidade, democraticidade e internacionalização. Os marcadores identificados resultam de estudos realizados no âmbito do projeto "Marcadores de formação, inovação e pesquisa para a Universidade de hoje e do próximo decénio" ${ }^{2}$, integrado e apoiado pelo

\section{CIDInE ${ }^{3}$.}

As sessões de reflexão e construção de cenários vêm na sequência da realização de entrevistas individuais a professores da Universidade de Aveiro, Portugal, e, em menor percentagem, a alguns professores de outras universidades portuguesas e brasileiras, bem como da aplicação de um questionário online (QuestionárioMarcadores: Pensar, sentir e organizar a Universidade do presente e do futuro), dirigido a professores, investigadores e estudantes da Universidade de Aveiro e de outras universidades brasileiras e portuguesas (Tavares, Oliveira, \& Alarcão, 2016).

\section{ENQUADRAMENTO TEÓRICO}

Partiu-se do pressuposto de que a instituição universitária, apesar de ser uma entidade multisecular, mudou bastante no decorrer dos tempos e está a mudar hoje mais rapidamente, mas talvez não tanto quanto seria necessário para poder corresponder à evolução da sociedade. Essa mudança e transformação, de acordo com os sinais que nos vão chegando, será mais intensa e profunda no futuro a curto, médio e longo prazo. Vários estudos têm sido realizados sobre a Universidade do futuro e, designadamente, sobre a Universidade dos próximos 10 ou 20 anos (Speller, Robl, \& Meneghel, 2012; Tavares, 2015; World Economic Forum, 2016). Alguns atrevemse até a olhar para períodos bastante mais longos, de 30, 50 ou 100 anos, mas, dada a velocidade que os acontecimentos configuram, ir para além de 20 ou 25 anos parece excessivo e dificulta a construção de cenários mais ou menos realistas que nos permitam antever, com um mínimo de previsibilidade, o futuro da Universidade em

\footnotetext{
${ }^{2}$ Informação adicional em: http://josetavares.wixsite.com/projeto-marcadores

${ }^{3}$ Centro de Investigação, Difusão e Intervenção Educacional (https://cidinegeral.wixsite.com/cidine), membro associado da EERA (European Education Research Association).
} 
geral e, sobretudo, estar preparados para agir com base num pensamento estratégico prévio flexível (na lógica de "se... então").

A abordagem através de cenários, e, designadamente, de cenários prospetivos, pareceu-nos ser uma metodologia adequada, promissora e desafiante para olhar para a instituição universitária dos próximos 20 anos e tentar antever o que poderá permanecer, transformar-se ou mesmo transmutar-se, em relação à produção do conhecimento, ao ensino e às aprendizagens, à relação com o exterior, às formas de comunicação e aos comportamentos dos seus principais atores. Não se trata de abordagens de recorte divinatório ou de tipo tarot, mas de saber ler o presente, ancorado na experiência do passado e nas janelas que, mais provavelmente, se abrirão no futuro.

É nesta linha que os teóricos das metodologias sobre a construção de cenários e a sua utilização nos estudos estratégicos e dinâmicas inteligentes sobre os países, as instituições e as organizações desenvolveram as suas teorias. Embora não seja nosso objetivo apresentar neste artigo as suas ideias e teorias, destacamos, entre outros, três autores que, aos nossos olhos, mais se distinguiram nesse campo: Michel Godet, Michael Porter, Peter Schwartz. Inicialmente utilizada no planeamento estratégico militar, esta abordagem depressa saltou para a indústria, a política e o desenvolvimento organizacional. Interessante para o nosso trabalho foi também verificar a crescente presença do fator pessoa no processo de planear o futuro, como se evidencia no título "People and Connections" (Shell International, 2002), relativo à criação de cenários, para 2020, pela Shell, uma das empresas que mais desenvolveu esta metodologia e que, tendo começado por se focalizar nos comportamentos dos preços do petróleo, cedo reconheceu a relevância das pessoas nas tomadas de decisão.

De entre as definições de cenário presentes na literatura, tomámos como referente a de Schwartz (cit. em Chermack, 2011): "A tool for ordering one's perception about alternative future environments on which one's decisions might be played out" (p. 14). Em termos operatórios, seguimos a linha do próprio Chermack (2011), para quem "scenario planning is a participative approach to strategy that features diverse thinking and conversation" ( p. 10).

Com base nos estudos que temos vindo a realizar no âmbito do projeto "Marcadores de formação, inovação e pesquisa para a Universidade de hoje e do próximo decénio", o nosso objetivo é a construção de cenários prospetivos sobre a Universidade, em geral, para os próximos 20 anos. Esses cenários assentarão na importância e intensidade que os diferentes marcadores, acima mencionados, poderão vir a assumir na estrutura e dinâmica da Universidade do futuro em relação à formação, à inovação e à pesquisa. Os marcadores por nós identificados foram descritos sucintamente para mais fácil compreensão e apresentados nesses mesmos termos aos participantes de cada uma das sessões com grupos focais, a saber:

1. As mentes (e o desejo de conhecer e aprender): Cérebros, talentos, inteligências brilhantes, espíritos lúcidos e equilibrados, QI (Quociente de Inteligência) acima da média, vontade e desejo de conhecer e aprender;

2. Os afetos (e o ambiente relacional): Sentimentos positivos nas pessoas e entre as pessoas, emoções, paixão, encorajamento, motivação, confiança, entusiasmo e envolvente positiva de trabalho e de estudo;

3. A autonomia: Capacidade de autoorientação e autodeterminação, livre e responsável, autoconfiança e autodomínio;

4. As tecnologias: Tecnologias da informação, da comunicação e da pesquisa (hardware e software);

5. Os métodos (de trabalho): Planeamento, 
gestão e organização dos processos de aprender, inovar e pesquisar dos professores, investigadores e estudantes;

6. A organização (institucional): Planeamento e gestão dos processos que dão estrutura à organização e servem os seus fins;

7. Os edifícios: Diferentes espaços dos campi (anfiteatros, salas, laboratórios, gabinetes, bibliotecas, cantinas, bares, espaços de convívio, residências, correio, bancos, espaços exteriores...);

8. Os financiamentos: Dotações de que a Universidade dispõe para a realização da sua missão na formação, inovação, pesquisa e extensão comunitária, social e cultural;

9. Os contextos: Ambientes físicos e psicológicos de trabalho e socialização que incluem os serviços disponibilizados pelos lugares e a envolvente socioeconómica e sociocultural;

10. Os equipamentos: Infraestruturas e materiais, instrumentos necessários à realização das atividades de pesquisa, ensino e inovação;

11. Os comportamentos: Dimensão psicológica, sociológica, cultural e ética das atitudes e do agir;

12. A empregabilidade: Capacidade de ser "empregável" e estar preparado para um trabalho múltiplo, diverso e flexível;

13. A democraticidade: Abertura ao maior número de estudantes sem perda de qualidade; participação inteligente, livre e responsável dos atores; facilidade de acesso à formação e ao conhecimento;

14. A internacionalização: Aceitação e participação transnacional e transcultural na construção e difusão do conhecimento científico, pedagógico, tecnológico e cultural.

\section{AS SESSÕES DE DISCUSSÃO E} R E F L E X Ã O

No sentido de conjugar a reflexão em grupo no processo de discussão e construção de cenários e o papel central que, nesse processo, as pessoas desempenham, decidiu-se interpelar os atuais atores do contexto universitário e fazê-lo, agora já não através de entrevistas e questionários, mas através da realização de workshops, em formato de grupo focal (Gray, 2004, p. 230), para potenciar oportunidade de diálogo, confronto de ideias e discussão, conjugados com momentos de reflexão individual. Privilegiouse uma abordagem construtivista, baseada na subjetividade, compreensão, dialogicidade, interatividade, contextualização, realidades múltiplas, indução e relativismo (Guba \& Lincoln, 1982, 1994). Tendo em conta os objetivos a alcançar, que serão explicitados mais à frente, optou-se por uma metodologia de construção de cenários, cuja apresentação, discussão e recolha de informações serão mais tarde trabalhadas.

Dado o número de participantes que se pretendia agregar (18 por sessão), o tempo de duração e interação (3 horas), a dinâmica que se pretendeu imprimir e os objetivos a alcançar, não se pode considerar ter-se tratado de grupos focais no sentido mais restrito do conceito (Barbour \& Kitzinger, 1999; CarliniCotrim, 1996; Greenbaum, 2000; Trad, 2009), mas de algo entre o grupo focal e o workshop ou sessão de trabalho. Por isso, a metodologia seguida não se confinou à que é normalmente utilizada nos grupos focais, mas situou-se entre duas dinâmicas: a do workshop e a do grupo focal, assente numa filosofia socioconstrutivista, participativa e implicada. As sessões realizaramse na Universidade de Aveiro, Portugal, em local apropriado e decorreram com dinâmicas, andamentos e materiais adequados para o efeito, como explicitaremos mais adiante. 


\subsection{PARTICIPANTES}

Os participantes foram professores, investigadores e estudantes, das várias áreas científicas (ciências exatas, engenharias, ciências sociais e humanas), individualmente convidados e em regime de voluntariado, num total de 51 , do sexo masculino e feminino, divididos em três sessões de 3 horas cada, realizadas em fevereiro e março de 2017. A distribuição teve em conta o equilíbrio dos participantes em cada uma das sessões no que respeita ao género e à categoria. Os participantes foram convidados, através de e-mail, pelo coordenador do projeto. Para chegar mais facilmente aos investigadores e aos estudantes, o coordenador solicitou aos Diretores das várias unidades orgânicas (departamentos e escolas) a indicação de nomes a convidar. Os professores foram convidados diretamente.

Para além destes participantes nos workshops, dinamizados e moderados pelo coordenador do projeto, estiveram também presentes cinco outros membros da equipa com funções de estimuladores da discussão, observadores/ comentadores com vista a preparar uma síntese final e tomadores de notas de contextualização relevantes para o posterior tratamento dos dados.

\subsection{OBJETIVOS}

Os objetivos definidos para estas sessões foram, essencialmente, os seguintes: a) criar oportunidades de reflexão em grupo sobre o tema em questão; b) confirmar ou infirmar a relevância dos marcadores apresentados; c) identificar cenários preferidos; d) avaliar a metodologia de cenários e e) recolher sugestões para a melhoria do modelo teórico subjacente.
2.3 A ORGANIZAÇÃO DAS SESSÕES NOS SEUS VÁRIOS ANDAMENTOS

As sessões foram pensadas e organizadas para serem tempos de interação, reflexão, discussão e trabalho, conducentes à troca de ideias e à recolha de informação. Na sua estruturação, foram identificados vários andamentos suportados por alguns materiais (Anexos 1 e 2). Assim, após uma nota introdutória, foram apresentados três cenários para discussão em momento de brainstorming livre. Intercalando momentos de discussão em grupo com momentos de reflexão individual, seguiu-se uma atividade de brainwriting 1. E, logo após esta atividade, novo momento de discussão, desta vez mais orientada para a defesa ou rejeição argumentativa dos cenários apresentados e preparatória da segunda atividade de escrita (brainwriting 2), com a construção do cenário individual por cada um dos participantes.

Antes de se dar início à sessão de brainwriting 2 realizou-se a atividade de registo individual de fundamentação da classificação da pertinência dos marcadores no cenário idealizado por cada um dos participantes. Este registo individual, em suporte de post-it (codificado e cartografado) sobre um "radar de marcadores" projetado na sala para todos os participantes (Figura 1) e recolhido para posterior tratamento, permitiu coligir, num suporte de perspetiva holística, a opinião de todos os participantes sobre todos os marcadores, conseguindo ainda fazê-lo respeitando uma escala valorativa de 1 a 10 de acordo com os argumentos de cada um (Figura 2). 


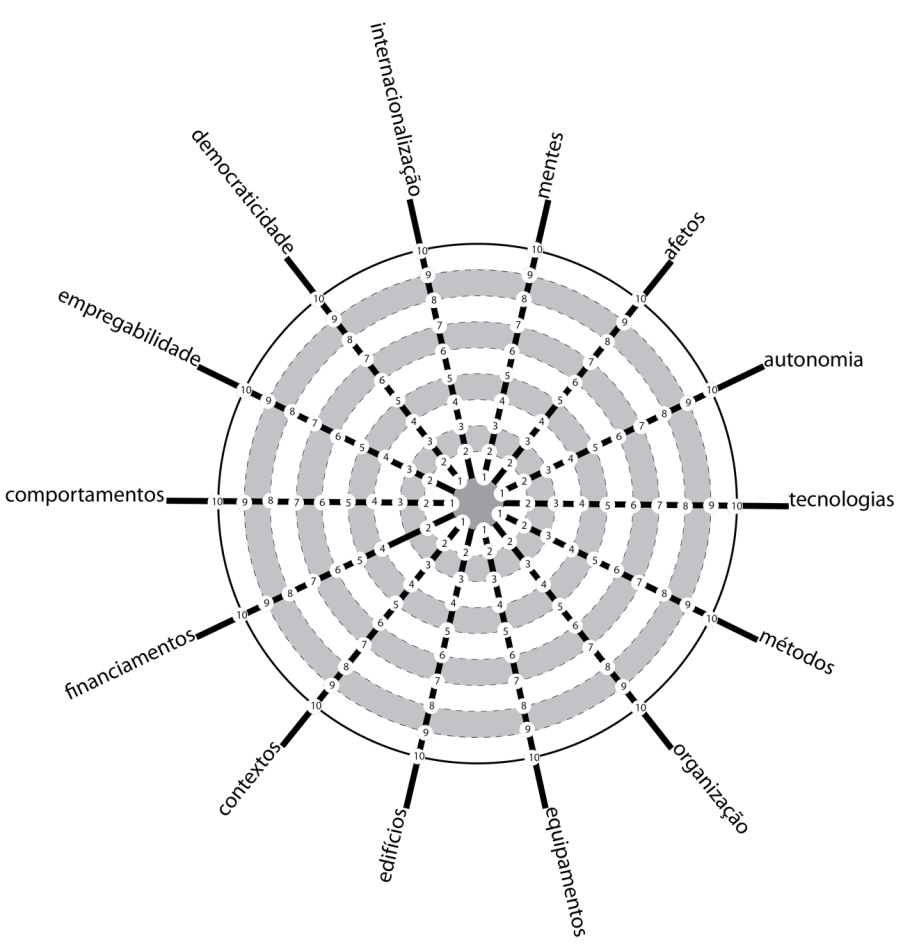

FIGURA 1 - "Radar de marcadores"

Esta atividade articulou-se com o brainwriting 2, um exercício de consolidação de pensamento e opinião de cada um dos participantes no contexto do desafio lançado.

A finalizar, os observadores/comentadores apresentaram os seus apontamentos e o coordenador explicou o que se tinha em mente para o desenvolvimento próximo do projeto, a saber: a) realização de uma sessão aberta e alargada sobre os resultados e conclusões obtidas nestas sessões (já realizada); b) reestruturação dos marcadores (já efetuada); e c) elaboração de artigos para publicação (dois já aceites para publicação).

As sessões foram gravadas em áudio e vídeo para posteriormente se poder analisar, completar, conferir e avaliar a informação recolhida, lançando mão de abordagens qualitativas e quantitativas ou mistas.

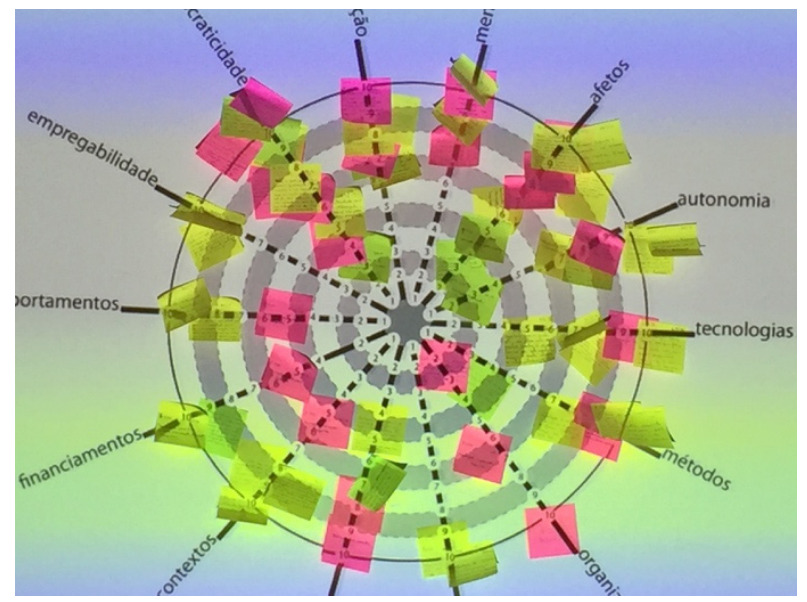

FIGU RA 2 - Scoreboard no radar de marcadores

2.4 A CONSTRUÇÃo dos CENÁrios. Dos CENÁRIOS APRESENTADOS AOS CENÁRIOS INDIVIDUALMENTE CONSTRUÍDOS

Como referido, este artigo tem vindo a focalizarse na metodologia de cenários prospetivos sobre a Universidade dos próximos 20 anos. Esses cenários foram construídos a partir da incidência e importância que os marcadores provavelmente virão a ter na estrutura e dinâmica da instituição universitária, em geral. Para a sua representação, foram criados cenários prováveis, com base nos valores atribuídos a cada um dos marcadores. Um dos cenários por nós apresentado (a que chamámos Cenário A) baseia-se, de certa forma, nos valores das médias obtidas a partir dos resultados das entrevistas e do questionário online acima referido (Tavares, Oliveira, \& Alarcão, 2016). Os outros dois cenários (B e C), um de recorte mais tradicional e autoritário e o outro mais utópico e laisser-faire, foram 
construídos por contraste com o cenário A, para maior "provocação" da discussão. Apresentamos a seguir os três cenários para a Universidade nos próximos 20 anos na sua forma descritiva e numa representação visual em radares (Figuras $3,4$ e 5$)$.

\section{Cenário A}

E se, na Universidade do futuro e, designadamente, na dos próximos 20 anos, se desenvolvesse, na formação, inovação e pesquisa, uma mentalidade mais competitiva, aberta, desafiadora, questionante, criativa, ética, descentralizada, cultural, autónoma, livre e responsável, o que aconteceria em relação aos marcadores mentes, afetos, autonomia, tecnologias, métodos, organização e gestão, edifícios e campi, financiamentos, contextos $e$ ambiente relacional, equipamentos, comportamentos, empregabilidade, democraticidade e internacionalização?

Então, às mentes, aos afetos, à autonomia, às tecnologias, à internacionalização e à empregabilidade seria dada uma forte relevância; os equipamentos, os métodos e a organização, os edifícios e os financiamentos continuariam a mobilizar especial atenção da direção da Universidade; os contextos, os comportamentos e a democraticidade seriam também bastante importantes, mas menos incisivos.

\section{Cenário B}

$E$ se, na Universidade do futuro e, designadamente, na dos próximos 20 anos, se seguisse e acentuasse, na formação, inovação e pesquisa, o ideário de uma mentalidade elitista, de "torre de marfim", distanciada, rígida, conservadora, centralizada, hierárquica, autoritária, subserviente, submissa, como seriam avaliados os marcadores mentes, afetos, autonomia, tecnologias, métodos, organização e gestão, edifícios e campi, financiamentos, contextos e ambiente relacional, equipamentos, comportamentos, empregabilidade, democraticidade e internacionalização?

Então, às mentes, aos métodos, à organização e gestão, às tecnologias e aos equipamentos e financiamentos seria dada uma grande importância; os afetos, a autonomia e a democraticidade teriam uma importância muito reduzida; os campi e os edifícios continuariam a mobilizar uma atenção especial por parte da direção da Universidade; a empregabilidade e a internacionalização teriam tendência a ser bastante valorizadas, por força da pressão interna e externa, bem como da competitividade e do prestígio da própria instituição.

\section{Cenário $C$}

E se, na Universidade do futuro e, designadamente, na dos próximos 20 anos, se desenvolvesse, na formação, inovação e pesquisa, uma atitude de abertura total, desestruturada, visionária, desafiadora, questionante, criativa, descentralizada, cultural, utópica, laisser-faire, o que aconteceria em relação às mentes, afetos, autonomia, tecnologias, métodos, organização e gestão, edifícios e campi, financiamentos, contextos e ambiente relacional, equipamentos, comportamentos, empregabilidade, democraticidade e internacionalização?

Então, às mentes, às tecnologias, aos afetos, à autonomia, aos equipamentos, à democraticidade, à internacionalização e à empregabilidade seria dada uma forte ou fortíssima relevância; os contextos, os edifícios, os financiamentos, os comportamentos, os métodos e a organização teriam uma importância diminuta. 


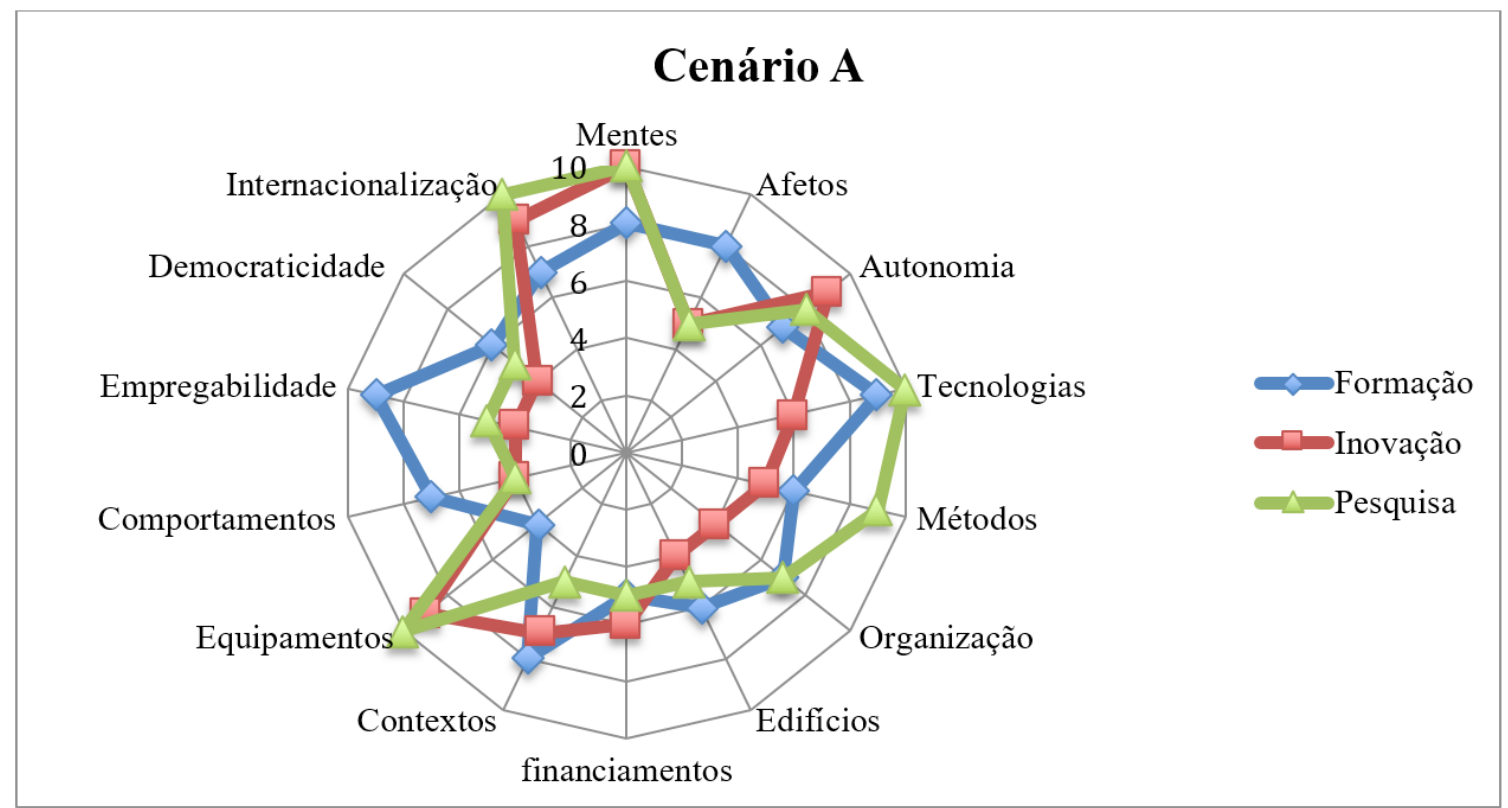

Fi gu R A 3 - Cenário A: Formação, inovação e pesquisa na Universidade dos próximos 20 anos

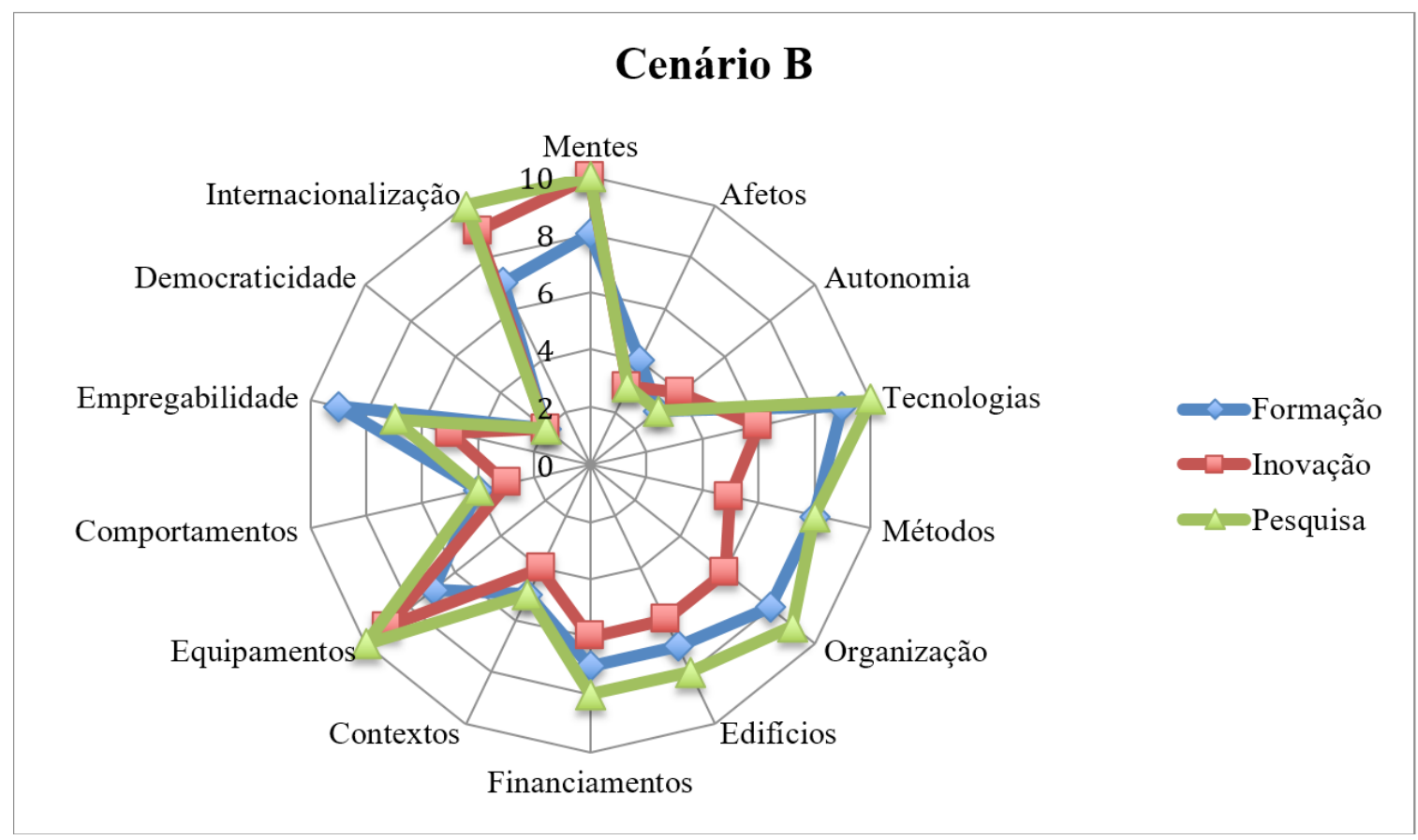

FI G URA 4 - Cenário B: Formação, inovação e pesquisa na Universidade dos próximos 20 anos 


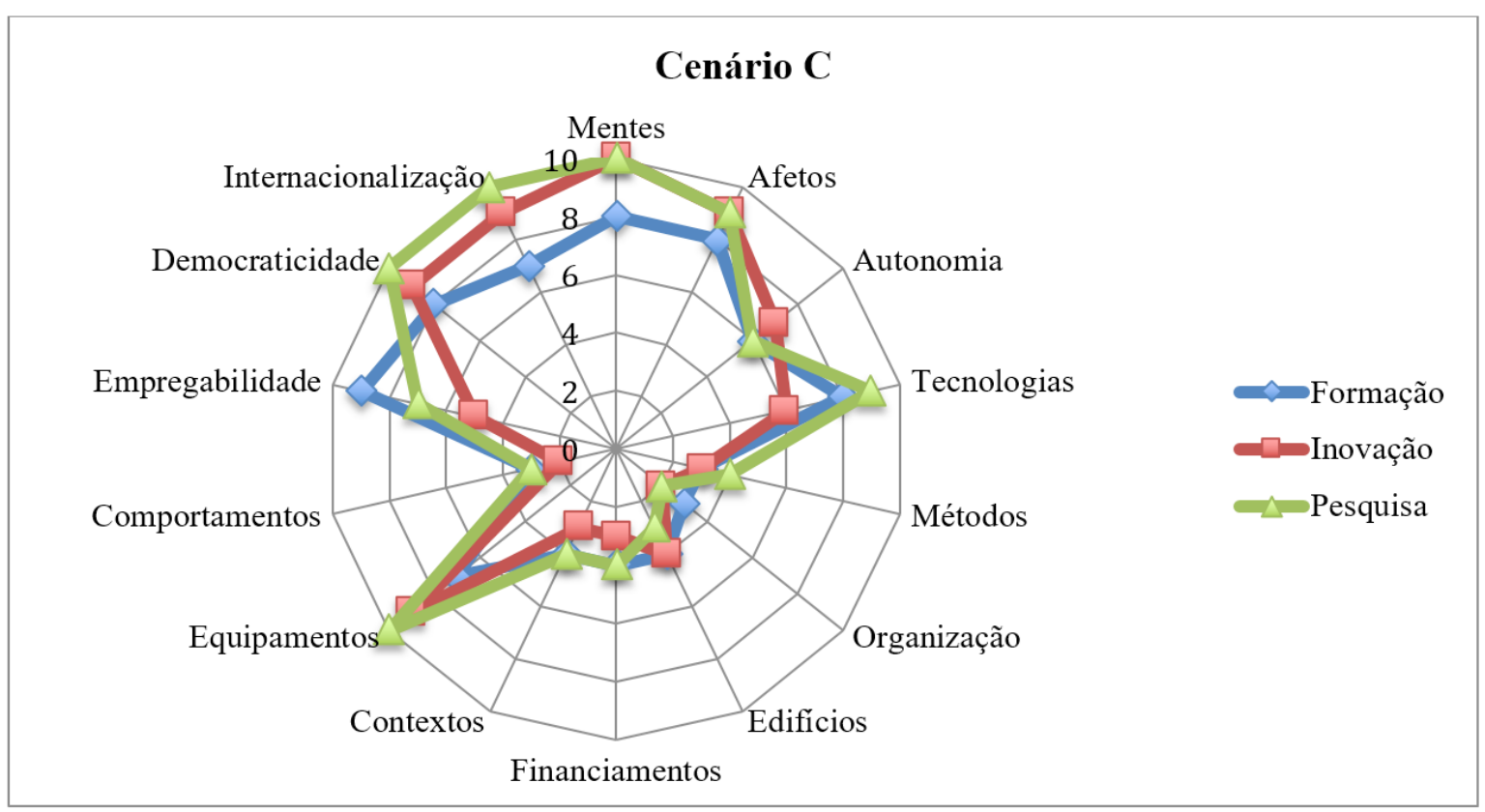

F I G U R A 5 - Cenário C: Formação, inovação e pesquisa na

Universidade dos próximos 20 anos

Foram estes os três cenários apresentados e debatidos nos workshops antes de os participantes serem convidados a construir os seus próprios cenários. Estes estão agora a ser analisados no que se refere ao contributo que podem trazer para o desenvolvimento do projeto "Marcadores de formação, inovação e pesquisa para a Universidade de hoje e do próximo decénio".

\subsection{RECOLHA DE INFORMAÇÃO E ANÁlise} DOS DADOS

A informação a tratar foi recolhida pelos seguintes processos: a) comentários dos observadores/anotadores; b) fichas preenchidas pelos participantes durante as duas atividades de brainwriting; c) fundamentação subjetiva associada a cada marcador num post-it associado a uma escala valorativa (scoreboard no radar de marcadores); e d) registos das sessões em áudio e vídeo.

Como expresso no resumo, neste artigo apresenta-se o resultado do tratamento dos dados referentes à abordagem utilizada, isto é, à base da discussão e construção de cenários em workshops/grupos focais ${ }^{4}$, incidindo assim sobre o objetivo d) (v. secção Objetivos) acima referido. Para o efeito, consideram-se as notas dos comentadores e as respostas dos participantes relativas a três questões colocadas nas atividades de brainwriting, a saber: "Já participou em sessões de reflexão sobre metodologia de cenários? Se sim, o que pensa dessa metodologia?" (questões 1 e 2 de brainwriting 1); "Acha que este esforço (de refletir e decidir sobre o cenário mais provável: $\mathrm{A}$, $\mathrm{B}, \mathrm{C}$ ) o fez refletir e foi útil? Justifique" (questão 5 de brainwriting 1); "Gostou da experiência? Sim/Não. Justifique" (questão 3 de brainwriting 2). Esta informação, de natureza qualitativa, foi trabalhada com o apoio do programa WebQDA.

\footnotetext{
${ }^{4}$ Uma reflexão sobre a pertinência dos marcadores irá ser apresentada no VII CIAIQ, 10 a 13 de julho de 2018, com a comunicação "Perspetivar a universidade a 20 anos a partir de um modelo de marcadores: A relevância da opinião", já aceite para integrar as atas do congresso, da autoria de O. Mealha, I. Alarcão, C. Almeida, J. B. Oliveira, J. Tavares, \& D. Souza - v. https://ciaiq. org/7o-congresso-ibero-americano-em-investigacao-qualitativa/
} 
$\mathrm{Na}$ opinião dos comentadores, as três sessões decorreram muito bem, com equilíbrio entre disciplina e espontaneidade e entre trabalho individual e partilha de opiniões. Os participantes mostraram-se, na sua grande maioria, interessados e participativos. Os estudantes, apesar de não usarem muito da palavra, mostraram-se empenhados nas tarefas individuais. A variedade das atividades parece ter contribuído para que as 3 horas de duração da sessão não fossem sentidas como excessivas. Uma das atividades que, pela sua novidade, introduziu uma certa dinâmica e boa disposição foi a colocação dos post-its no scoreboard em radar de marcadores, "holístico", projetado na sala.

As respostas dos participantes às questões acima enunciadas revelaram que apenas nove tinham alguma experiência de participação em sessões com metodologia de cenários, sendo que quatro a qualificaram como uma dinâmica desafiadora. Nas respostas à questão 5 do brainwriting 1, relativa à utilidade do questionamento sobre diferentes cenários, contabilizaram-se 65 referências com destaque para o reconhecimento de uma reflexão oportuna e pertinente (30 referências). Um investigador registou a abertura de horizontes, ao escrever: "Existem indicadores propostos que parecem ter uma importância grande na universidade e sobre os quais não tinha pensado antes, nomeadamente os afetos e os contextos". Um professor comentou: "O facto de aceder a perspetivas diversas provocou a minha reflexão, ponderando outras ideias, confrontando com as que tinha previamente... E daí resultam sempre algumas mudanças e enriquecimento". Foi também salientada a valorização da opinião pessoal (10 referências), de que é exemplo a expressão de um estudante ao afirmar: "porque fez-me pensar e refletir sobre o que é fundamental para uma universidade e demonstrou a minha perspetiva de uma forma que pode vir a ser útil”. Questionados, no final, sobre se tinham gostado das sessões, todos os participantes, exceto dois, responderam afirmativamente, destacando a oportunidade de reflexão e partilha que lhes foi proporcionada. Nas palavras de um dos professores, "é sempre motivante e enriquecedor discutir a universidade que queremos com outros membros da comunidade académica tão diversos (em áreas, perfis, idade, pertenças e formação académica)". Um investigador comentou que foi "promotor de uma reflexão sobre o papel individual que cada um de nós desempenha naquilo que é a universidade de hoje e um papel que pode desempenhar naquilo que será a universidade de amanhã. Devemos assumir a nossa responsabilidade individual em todo este processo e desenvolvimento da própria universidade". Outro investigador expressava a sua satisfação ao afirmar: "Muito interessante, sobretudo pela partilha de ideias e por me fazer pensar 'out of the box"'. Os dois participantes que não gostaram (estudantes) apresentaram as seguintes justificações: "não foi ao encontro das minhas perspetivas e penso que o estudo e as sessões podiam ter outros pontos de foco e deviam ser mais concisas e objetivas"; “demasiadas pessoas para uma duração tão pequena”.

\section{CONSIDERAÇÕES FINAIS SOBRE O MODO COMO DECORRERAM AS SESSÕES}

Neste artigo, foram desenvolvidos apenas os processos metodológicos e organizacionais utilizados em três sessões de grupos focais em formato de workshop destinadas a refletir sobre o cenário que, mais provavelmente, configurará a Universidade, como instituição, nos próximos 20 anos. Nestes comentários finais, tentaremos identificar os pontos fortes e fracos, as facilidades e dificuldades que encontrámos, as limitações que reconhecemos, as oportunidades que se abriram e os contributos que foram trazidos para o desenvolvimento do projeto "Marcadores 
de formação, inovação e pesquisa para a Universidade de hoje e do próximo decénio".

Comecemos pelas facilidades/dificuldades. A descrição dos cenários apresentados não ofereceu grande dificuldade, uma vez que se partiu de um cenário hipotético, construído com base no trabalho empírico feito numa fase anterior do projeto, através de entrevistas e questionários, e que depois se elaboraram os outros dois cenários por contraste. $O$ formato das sessões também não se revelou difícil quanto à sua operacionalização, já que os materiais foram previamente preparados e estavam adequados aos objetivos que se pretendia atingir. $\mathrm{O}$ nível de interesse e colaboração dos participantes foi um aspeto muito positivo, encorajador e facilitador. A maior dificuldade sentiu-se na constituição dos grupos dos professores, investigadores e estudantes a convidar, não só na sua identificação, como também na harmonização de horários. Neste aspeto foi muito útil a colaboração dos diretores dos departamentos e escolas, como acima referimos. Esta tarefa teria sido facilitada se tivéssemos optado por grupos isomórficos de professores, investigadores ou estudantes, mas o que se perdeu em trabalho ganhou-se em riqueza de vários pontos de vista e maior democraticidade. De registar ainda o contributo positivo que o envolvimento dos diretores dos departamentos, escolas e elementos de governo da Universidade trouxe, no que respeita à aceitação e divulgação do projeto.

Como em geral nas investigações, também nesta nem tudo é perfeito, e uma das limitações a ter em conta é o facto de se tratar de uma amostra reduzida, não sendo por isso possível generalizar que projeção faz a comunidade académica relativamente à Universidade do futuro. Contudo, entendemos que este processo foi capaz de sublinhar evidências de opinião e apontar possíveis cenários através da convergência de opiniões em determinadas perspetivas e conjuntos de marcadores, uma probabilidade que só um tratamento mais aprofundado da informação recolhida poderá confirmar ou infirmar. Mesmo que se venham a confirmar estas nossas perspetivas, sabemos bem como qualquer exercício prospetivo desta natureza está sempre ameaçado, pois não consegue prever variáveis e alterações contextuais internas ou vindas do exterior do ecossistema em causa e que podem alterar completamente os pressupostos da projeção. Mas, ainda que ponderando as situações de "ameaça", se o processo de reflexão e prospeção de cenários estiver correto, a reorganização de um "cenário futuro", no advento de situações imponderáveis sobre a Universidade, será muito mais eficaz.

Finalmente, salientamos a oportunidade que este exercício proporcionou aos participantes de, individualmente e em grupo, num ambiente socioconstrutivo, refletirem sobre o que poderá ser a Universidade do futuro numa visão a médio prazo e quais as implicações nas dimensões de formação, de inovação e de pesquisa. Aos membros do projeto forneceu a oportunidade de repensarem alguns dos marcadores apresentados, de que resultou: aglutinação de "tecnologia" com "equipamentos", "métodos" com "organização", "edifícios" com "contextos"; introdução do marcador "sustentabilidade" e nova descrição de "contexto".

A conceção e estruturação das sessões e os materiais para elas elaborados constituem uma oportunidade para que o processo possa ser replicado noutros contextos de intervenção, junto da comunidade universitária, que conduzam a uma maior consciencialização acerca da Universidade do futuro e a novas formas de atuar.

\section{REFERÊ NCIAS}

Barbour, R. S., \& Kitzinger, J. (1999). Developing focus group research. London: Sage.

Carlini-Cotrim, B. (1996). Potencialidades da técnica qualitativa grupo focal em investigações sobre abuso de substâncias. Revista de Saúde 
Pública, 30(3), 285-293.

Chermack, T. J. (2011). Scenario planning in organizations. How to create, use and assess scenarios. Oakland: Berrett-Koehler Publishers.

Damásio, A. (1994). Descartes' error: Emotion, reason, and the human brain. New York: Grosset/Putnam.

Damásio, A., Tranel, V., \& Damásio, H. (1991). Somatic markers and the guidance of behavior: Theory and preliminary testing. In H. S. Levin, H. M. Eisenberg, \& A. L. Benton (Eds.), Frontal lobe function and dysfunction (pp. 2017-2029). New York: Oxford University Press.

Gray, D. E. (2004). Doing research in the real world. London: Sage.

Greenbaum, T. L. (2000). Moderating focus groups: A practical guide for group facilitation. Thousand Oaks: Sage.

Guba, E. G., \& Lincoln, Y. S. (1982). Epistemological and methodological bases of naturalistic inquiry. Educational Communication and Technology: A Journal of Theory, Research and Development, 4(30), 233-252.

Guba, E. G., \& Lincoln, Y. S. (1994). Competing paradigms in qualitative research. In N. K. Denzin \& Y. S. Lincoln (Eds.), Handbook of qualitative research (pp. 105-117). Thousand Oaks: Sage.

Moritz, G., Pereira, M., Souza, I., Herling, L., Moritz, M., \& Cesconetto, S. (2010). A prospecção de cenários nas universidades: Variáveis portadoras de futuro $e$ a trajetória estratégica da UFSC para 2022. Disponível em: https://repositorio.ufsc.br/ bitstream/handle/123456789/97033/A\%20 P R O S P E C \% C $3 \% 87 \%$ C $3 \% 83$ O \% 20 D E \% 20 C E N \% C $3 \% 81$ R I O S \% 20 NAS \% 20 U N I VER S I A D E S \% 20 VARI \% C $3 \% 81$ VEIS \% 20 P ORTA D O. pdf? sequence $=1$ (acesso em: 05/03/2017).

OCDE/CERI. (2004). Four future scenarios for higher education. The university future project. Disponível em: http://www.oecd.org/ education/skills-beyond-school/ceri-universit yfuturesfourscenariosforhighereducation.htm (acesso em: 14/11/2016).

Shell International (2002). Exploring the future. People and connections: Global scenarios to 2020. Public summary disponível em: https:// www.pik-potsdam.de/avec/peyresq2005/ talks/0921/leemans/literature/shell_global_ scenarios.pdf

Speller, P., Robl, F., \& Meneghel, S. M. (Orgs). (2012). Desafios e perspectivas da educação superior brasileira para a próxima década. Brasília: UNESCO, CNE, MEC.

Tavares, J. (2015). Marcadores de formação, inovação e pesquisa para a Universidade do próximo decénio. Palestra apresentada no IX Colóquio Internacional "Educação e Contemporaneidade", 17 a 19 de setembro de 2015, São Cristóvão, SE, Brasil. Disponível em: https://www.researchgate. net/publication/310425410_Marcadores_ de_formacao_inovacao_e_pesquisa_para_a_ Universidade_do_proximo_decenio (acesso em: 17/07/2016).

Tavares, J., Oliveira, J., \& Alarcão, I. (2016). Marcadores de formação para a universidade de hoje e do próximo decénio: Primeiros passos de um projeto de pesquisa. Revista Transmutare, 2(1), 214-253. doi:10.3895/rtr. v1n2.4982

Trad, L. B. (2009). Grupos focais: Conceitos, procedimentos e reflexões baseadas em experiências com o uso da técnica em pesquisas de saúde. Physis: Revista de Saúde Coletiva, 19(3), 777-796. doi:10.1590/S010373312009000300013

UNESCO. (2003). Challenges of the university in the knowledge society, five years after the World Conference on Higher Education. Paris: UNESCO.

World Economic Forum. (2016). Future of jobs. Disponível em: http://reports.weforum.org/ future-of-jobs-2016/ (acesso em: 30/11/2017). 
THE UNIVERSITY FOR THE NEXT TWENTY YEARS: A VIEW BASED ON A SCENARIO METHODOLOGY

\section{Abstract}

In this paper the authors propose to report the process and analyse the results of a scenario-based methodological approach to perceive prospective views for the future of the University in what concerns to education, innovation and research and according to the value intensity given to a set of markers: minds, affects, autonomy, technologies, methods, organization, buildings, funding, contexts, equipments, behaviours, employability, democraticity and internationalization, under study in the Project "Markers of education, innovation and research for the University of today and of the next decade". The focus of the present text is not the content under discussion, i.e. the prospective view of the evolution of the university in the future; it focuses on the methodology of construction of prospective scenarios in sessions with professors, researchers and students. The methodology of the sessions combined the dynamics of workshop and focus groups. The discussion of results focuses on difficulties, potentialities and limitations of the approach, as well as contributions for the global study.

KeY-wORDs: University; Scenarios; Focus groups; Markers
Pensar La UNiVERSIDAd DE LOS PRÓXIMOS 20 AÑOS A TRAVÉS DE UNA METODOLOGÍA DE ESCENARIOS

\section{RESUMEN}

En este artículo, los autores se proponen relatar el proceso y analizar los resultados de un enfoque metodológico basado en la construcción de escenarios para perspetivar la Universidad del futuro en las dimensiones de la formación, innovación e investigación y en función de la intensidad valorativa de un conjunto de marcadores: mentes, afectos, autonomía, tecnologías, métodos, organización, edificios, financiamientos, contextos, equipos, comportamientos, empleabilidad, democraticidad $y$ internacionalización, en estudio en el Projeto "Marcadores de formación, innovación e pesquisa para la Universidad de hoy y del prójimo decenio". El presente texto no incidirá sobre la substancia en estudio, es decir, cómo se ve la evolución de la Universidad en el futuro, pero sobre la metodología de construcción de escenarios prospectivos en sesiones que combinaron una dinámica de taller con la de grupo focal envolviendo a profesores, investigadores y estudiantes. Analizaremos las dificultades y potencialidades de este abordaje, las limitaciones encontradas y los aportes para el estudio global.

Palabras-clave: Universidad; Escenarios; Grupos focales; Marcadores

I Centro de Investigação “Didática e Tecnologia na Formação de Formadores” (CIDTFF), Universidade de Aveiro, Portugal. ORCID: 0000-0001-5356-0931

"Departamento de Educação e Psicologia, Universidade de Aveiro, Portugal. ORCID: 0000-0003-1391-5819

III Departamento de Comunicação e Arte, Centro de Investigação DigiMedia,Universidade de Aveiro, Portugal. ORCID: 0000-0002-4564-0496

Iv Centro Universitário Adventista de São Paulo (UNASP), Brasil \& Centro de Investigação "Didática e Tecnologia na Formação de Formadores” (CIDTFF), Portugal. ORCID: 0000-0003-3294-8624 


\section{ANEXO 1}

\section{Brainwriting 1}

(folha de respostas)

\section{Código}

Instituição universitária: Género: M $\mathrm{F} \_$Idade:

Tempo de serviço: Ano de curso: $\mathrm{L}$ $\mathrm{D}$ Anos de pesquisa:

Área científica:

Responda, por favor, às seguintes questões:

1. Já participou em sessões de reflexão sobre metodologias de cenários? Sim Não

2. Se sim, o que pensa dessa metodologia?

3. Qual dos cenários apresentados, acha mais provável para a Universidade dos próximos 20 anos:

A , B , C

4. Porquê?

5. Acha que este esforço o fez refletir e foi útil? Sim Não Justifique

Data ---------/----

\section{Obrigado}




\section{ANEXO 2}

\section{Brainwriting 2}

(folha de respostas)

\section{Código}

Instituição universitária: Género: M F___ Idade:

Tempo de serviço: Ano de curso: $\mathrm{L}$ M D Anos de pesquisa:

Área científica:

\section{Responda às seguintes questões:}

1. Mantém ou altera a sua opinião em relação ao cenário por que optou para a Universidade dos próximos 20 anos? Se altera, diga porquê e em que marcadores.

2. Observe os radares das figs. 1, 2 e 3 e coloque um $X$ nas pontuações de 1 a 10 (1 mínimo e 10 máximo) dos marcadores de acordo com o cenário que espera como o mais provável para a Universidade dos próximos 20 anos. Neste contexto, as figs. 1, 2 e 3 apresentavam o radar da figura 1, no texto, para recolha das valorações incidindo sobre: formação, inovação e pesquisa

3. Gostou da experiência? Sim Não

4. Justifique:

Data ----/----/----

\section{Obrigado}

